\title{
Generation of Super-Radiance rf Pulses in a Sectioned Backward-Wave Oscillator
}

\author{
A.V. Savilov* \\ Institute of Applied Physics, Russian Academy of Sciences, 46 Ulyanov St., Nizhny Novgorod, 603950, Russia
}

\begin{abstract}
This work develops the theory of a backward-wave electron oscillator, which operates in the regime of the socalled super-radiation of short powerful rf pulses. According to simulations, the sectioning of the electron-wave interaction region can provide a significant enhancement of the power of the output rf pulse in relatively short systems.
\end{abstract}

\section{INTRODUCTION}

An effective method for generation of powerful pulses of coherent microwave electromagnetic radiation is the use of the so-called super-radiance (SR) regime of operation of a Cherenkov backward-wave oscillator (BWO) [1-9]. It is well-known that if the length of the electron-wave interaction region of a BWO significantly exceeds the starting threshold, then the transient process of excitation of the oscillator includes excitation of a short powerful rf pulse [1]. The peak power of this pulse is significantly higher than a characterictic output rf power in the regime of the stationary generation of the BWO. Moreover, the instantaneous efficiency of the generation of such rf pulse (the ratio of the rf pulse peak power to the power of the electron beam, which is also called "conversion factor") may exceed $100 \%$. This fact has been observed in experiments $[6$, 8 ], and it does not contradict the energy conservation law. Actually, the process of the emission of the SR rf pulse has a principally nonstationary character, namely, a relatively short backward (counter-propagated) rf pulse is formed in the process of its motion via a significantly longer electronbeam pulse. As a rule, a characteristic energy efficiency of this process does not exceed $20 \%$.

It should be noticed that the $100 \%$ level of the compression factor can be exceeded only in systems with a special optimization of the electron-wave interaction region; namely, profiling of corrugation depth of the operating waveguide is used [5]. According to simulations, this should provide a significant enhancement of the peak power of the pulse; however, this method requires a considerable increase of the system length. Because of the latter, the process of super-radiation becomes significantly more sensitive to the influence of the spread in electron velocity and of the frequency dispersion of the group wave velocity. Evidently, this is the reason that the conversion factors achieved in SR BWO experiments are still far from the most optimistic theoretical predictions.

*Address correspondence to this author at the Institute of Applied Physics, Russian Academy of Sciences, 46 Ulyanov St., Nizhny Novgorod, 603950, Russia; E-mail: savilov @ appl.sci-nnov.ru
This brief paper proposes a different method of the optimization of the operating region, which is based on sectioning of the electron-wave coupling factor and is aimed to enhancement of the peak power of output rf pulses of a SR BWO. The main advantage of this method is that the significant increase of the power of the SR rf pulse can be achieved at relatively short lengths of the operating waveguide.

The paper is organized as follows. Section 2 describes the set of 2D spatio-temporal equations of a BWO. Section 3 is devoted to numerical simulations of a uniform BWO, as well as of an auto-oscillator with various types of smooth profiling of the electron-wave coupling factor. In Section 4 a sectioned SR BWO is studied.

\section{SPATIO-TEMPORAL EQUATIONS OF A BACKWARD-WAVE OSCILLATOR}

Let us consider the simplest modes of a Cherenkov BWO, when a tubular rectilinear electron beam interacts with a backward rf wave (Fig. 1). The corrugation of the wall of the operating cavity provides a slow spatial harmonic of the rf wave; the axial component of its electric field has the following form:

$E_{z}=\mu \operatorname{Re} A(z, t) \exp \left(i \omega_{0} t-i h_{0} z-i \bar{h} z\right)$,

where $A(z, t)$ is the slow amplitude of the wave, $\bar{h}$ is the corrugation wavenumber, the frequency and the axial wavenumber in the wave phase are determined by the exact Cherenkov electron-wave resonance condition $\omega_{0}=\left(h_{0}+\bar{h}\right) V$ (here $V$ is the electron velocity and $h_{0}=h\left(\omega_{0}\right)$ is the axial wavenumber at the synchronous frequency, $\left.\omega_{0}\right)$, and the coupling factor $\mu$ is determined by the corrugation depth. In the case of a sectioned system, the resonant electron-wave interaction is absent in the middle smooth-wall section of the operating waveguide.

Spatio-temporal dynamics of excitation of the BWO is described by the following equation for the normalized amplitude of the rf wave [10], which is obtained in the approximation of the absence of the frequency dispersion of the group wave velocity: 


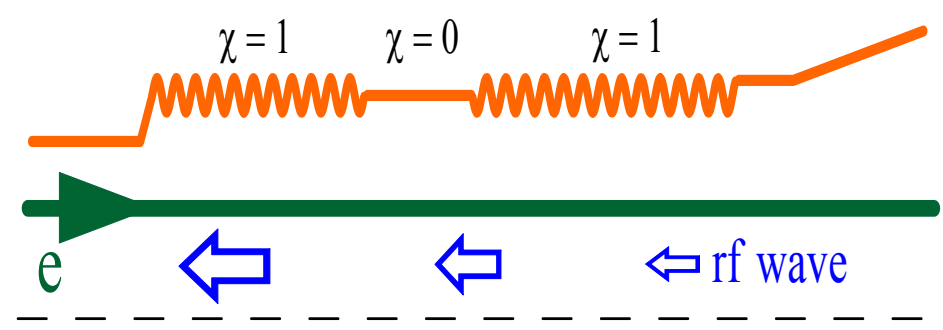

Fig. (1). Schematic of a Cherenkov BWO with sectioned electron-wave coupling factor, $\chi$.

$\frac{\partial a}{\partial \tau}-\frac{\partial a}{\partial \zeta}=\frac{\chi(\zeta)}{\pi} \int_{0}^{2 \pi} \exp (-i \theta) d \theta_{0}$

The right-hand part (the "source") of this equation should be found from equations of the electron motion. If the electron-wave interaction causes a relatively small change of the electron energy, then these equations have the form of a mathematical pendulum [11]:

$\frac{\partial u}{\partial \zeta}=\chi(\zeta) \operatorname{Re} a \exp (i \theta), \frac{\partial \theta}{\partial \zeta}=u$.

These equations have been normalized to the Pierce amplification parameter [11],

$C=\sqrt[3]{\mu^{2} v G}$

where $v=1 / \beta_{0}^{3} \gamma_{0}^{3}$ is the factor of inertial electron bunching (with $\beta_{0}=V_{0} / c$ and $\gamma_{0}=1 / \sqrt{1-\beta_{0}^{2}}$ ), and $G \propto I / N$ is the wave excitation parameter proportional to the electron current and reversely proportional to the wave norm. Correspondingly, $\tau=C \omega_{0} t$ and $\zeta=C \omega_{0} z / c$ are the normalized time and the axial coordinate, $u=\left(\gamma_{0}-\gamma\right) v / C$ is the normalized change of the electron energy, $\theta=\omega_{0} t-h_{0} z-\bar{h} z$ is the electron phase with respect the resonant spatial harmonic of the wave, and $a=\left(e A / \omega_{0} m c\right)\left(\mu v / C^{2}\right)$ is the normalized amplitude of the rf wave. form:

The initial conditions for electrons have the following

$u(\zeta=0)=0, \quad \theta(\zeta=0)=\theta_{0}$,

where the initial phases, $\theta_{0}$, are distributed uniformly over the interval $[0,2 \pi]$. The boundary condition for the amplitude of the backward rf wave,

$a(\zeta=L)=0$,

corresponds to the situation when there is no rf signal at the output of the operating waveguide, $\zeta=L$. In Eqs. (1) and (2), function $\chi(\zeta)$ describes sectioning of the factor of the electron-wave coupling, which is provided by sectioning of the corrugation depth (Fig. 1); in the simplest case of the uniform non-sectioned system $\chi=1$.

The normalized power of the wave is determnied by the following relation: $p=|a|^{2} / 4$.

In accordance to the energy conservation low, in the regime of the stationary single-frequency operation of the BWO the normalized output rf power coincides with the normalized electron efficiency:

$p(\zeta=0, \tau)=\frac{1}{2 \pi} \int_{0}^{2 \pi} u(\zeta=L, \tau) d \theta_{0}$.

Let us notice that the set of equations (1)-(4) does not include an electron-current parameter, as it has been normalized to the Pierce amplification parameter $C \propto \sqrt[3]{I}$, which is proportional to the cubic root of the electron current [11]. Thus, the normalized length of the system is proportional to the real length and the Pierce parameter:

$L=\frac{\omega_{0}}{c} C \propto l \sqrt[3]{I}$.

Within frameworks of the set (1)-(4), this normalized length is the only parameter determining dynamics of the excitation of a uniform BWO. The auto-oscillator is excited if the starting threshold $L_{\mathrm{st}} \approx 2$ is exceeded. When going slightly beyond this threshold, the resulting BWO regime corresponds to a stationary rf generation with a characteristic normalized output power $p \sim 1$ (Fig. 2, $L=2.7$ ). The increase of the normalized length, $L$, leads to a transition to regimes with periodical auto-modulations of the output $\mathrm{rf}$ power (Fig. 2, $L=3.5$ ).

The further increase of $L$ results in a complication of the modulation character of the output $\mathrm{rf}$ power, $p(\tau)$. In this case, the highest power is achieved in the first peak of these modulations (Fig. 2, $L=4.5$ ); and this power is significantly higher than a characteristic power of the stationary generation of the BWO. The principle of operation of a SR BWO is based on generation of the powerful SR rf pulse, occurring when the normalized threshold length and thus the starting current are significantly exceeded.

\section{SR BWO WITH A SMOOTH PROFILING OF THE ELECTRON-WAVE COUPLING FACTOR}

Fig. (3) illustrates results of optimizations of the SR BWO with various types of profiling of the electron-wave coupling factor, $\chi(\zeta)$, namely, the uniform system, the case of a linear profiling of the coupling factor, and the case of a sectioned system. These are results of self-consistent (PIC) simulations of Eqs. (1)-(4). 


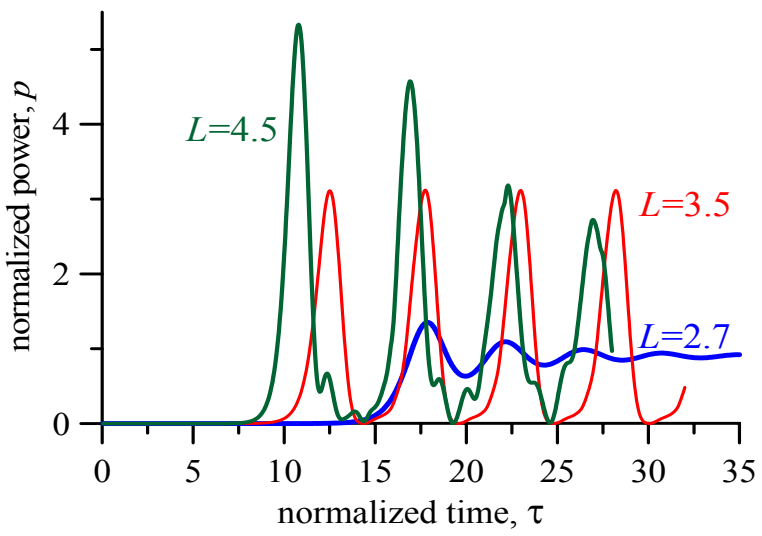

Fig. (2). The uniform BWO. Normalized output power versus the time at various normalized lengths of the electron-wave interaction regions.

In the simplest case of the uniform system $(\chi=1)$, the maximal power of the SR rf pulse is achieved at $L=7$ and amounts to $p \approx 8$ (Fig. 3, point 1 ). A natural and wellstudied [5] method for enhancement of the output power of a SR BWO is the use of a smooth profiling of the electronwave coupling factor $\chi(\zeta)$. Fig. (3) illustrates the results of simulations for the case of a simple linear profiling (curve 2 in Fig. 3)

$\chi(\zeta)=1-\alpha+\alpha \zeta / L$

as well as for the case of a more complicated profile $\chi(\zeta)$, when the electron-wave coupling factor is uniform in the first section of the operating waveguide, and increases linearly in the second section (curve 3 in Fig. 3):

$\chi(\zeta)=\left\{\begin{array}{lr}1-\alpha, & 0<\zeta<L / 2 \\ 1-\alpha+\alpha(\zeta-L / 2) /(L / 2), & L / 2<\zeta<L\end{array}\right.$.

The main idea of this approach can be formulated as follows. In order to enhance the peak power of the SR rf pulse, one should increase the number of electrons which follow via the counter-propagated rf pulse and pass their energy to this pulse. Evidently, this can be provided by increasing the length of the operating waveguide. However, the length should not be too long as compared to the starting threshold. Actually, the situation should be avoided when at some location within the BWO a separated oscillator is excited. In this situation the generation of the "operating" SR pulse (which follows through the whole region of the electron-wave interaction) is "spoiled" due to generation of a "parasitic" SR pulse in an input part of the operating waveguide (Fig. 4). In order to avoid this situation, the electron-wave coupling should become weaker in the input part of the BWO, which can be provided by profiling the coupling factor $\chi(\zeta)$. Thus, the profiling is needed to achieve the following aim: the normalized lenght of the system should be increased together with the increase of the starting length threshold.

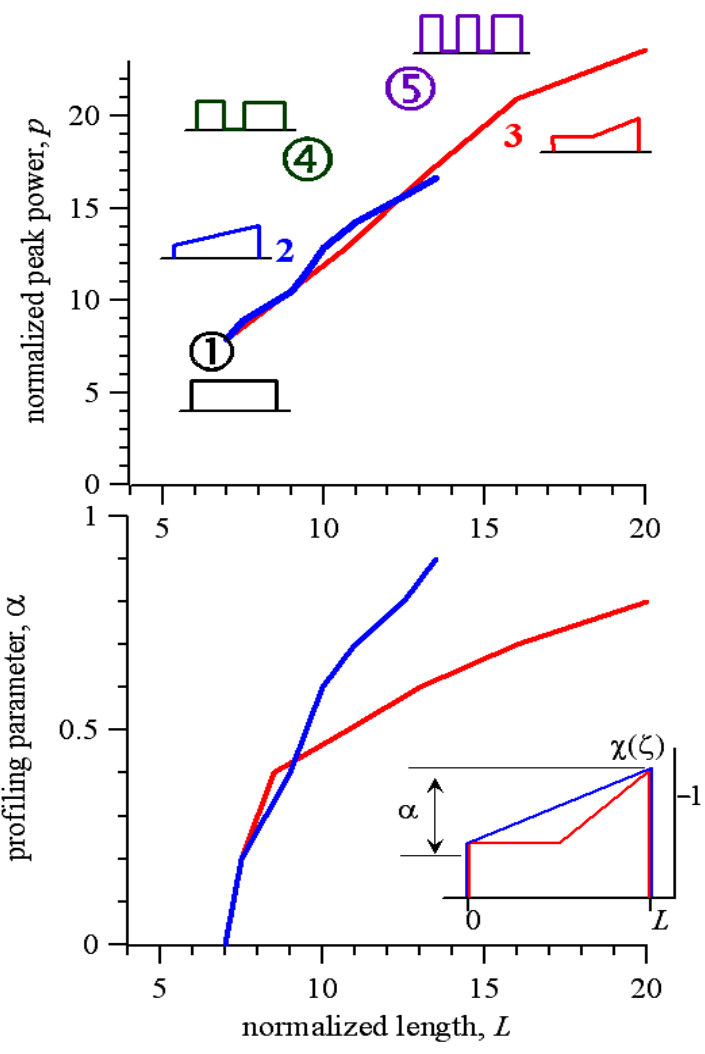

Fig. (3). Normalized output peak power of the SR BWO versus the optimal normalized length of the system: point 1 corresponds to the uniform system, curves 2 and 3 correspond to BWOs with simple [Eq.(5)] and sectioned [Eq. (6)] linear profiling of the electronwave coupling factor, whereas points 4 and 5 illustrate the twostage and three-stage sectioned BWOs. The lower plot illustrates the dependences of the profiling parameter on the optimal normalized length, as well as the coupling factor profiles $\chi(\zeta)$ in the cases of linear profilings Eq.(5) (blue curves) and Eq. (6) (red curves).

The main disadvantage of this method is caused by a weak electron-wave interaction in the input part of the operating cavity. Due to this fact, a significant increase of the lenght of the operating waveguide is needed to achieve a considerable enhancement of the output rf power. Therefore, the process of super-radiation can become more sensitive to the influence of the spread in electron velocity and of the frequency dispersion of the group wave velocity. According to simulations (Fig. 3, curves 2 and 3), the output rf power is approximately proportional to the optimal length of the operating waveguide. As compared to the uniform BWO (point 1 in Fig. 3), the simple linear profiling [Eq. (5)] of the electron-wave coupling factor $\chi(\zeta)$ (curve 2 in Fig. 3) can provide twice an enhancement of the output $\mathrm{rf}$ power ( $p \approx 16.6$ ); however, the optimal length of the operating waveguide $L=13.5$ is also nearly twice as long as the optimal length in the uniform case. In the case of the "sectioned linear" profile Eq. (6) (Fig. 3, curve 3) the output 
power is increased in three times $(p \approx 23.6)$, whereas the optimal length of the system $(L=20)$ is also nearly three time longer than that in the uniform case.

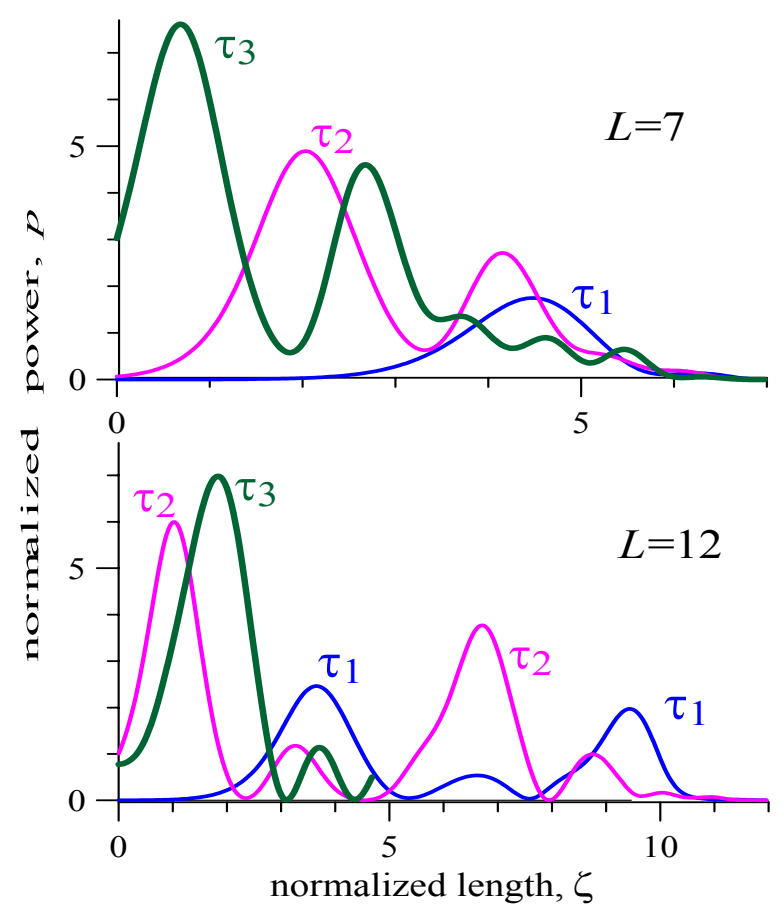

Fig. (4). The uniform BWO with nornalized lengths $L=7$ and $L=12$. Spatio-temporal evolution of the generation of the SR $\mathrm{rf}$ pulse (axial distributions of the normalized rf power inside the electronwave interaction region in various moments of the time, $\tau$ ). In the case of $L=12$, the "operating" SR pulse is "spoiled" by the "parasitic" SR pulse (whose generation begins in the middle of the interaction region).

Fig. (5) illustrates shapes of output rf pulses in the optimal regimes of SR BWOs with the uniform interaction region (pulse 1), as well as with simple [Eq. (5)] and sectioned [Eq. (6)] smooth profiling of the electron-wave coupling factor (pulses 2 and 3, respectively). At a fixed initial rf noise, the increase of the length of the autooscillator results in the increase of the time required to generate the SR rf pulse. At the same time, the duration of the $\mathrm{rf}$ pulse is almost the same in all regimes.

\section{SECTIONED SR BWO}

This work proposes a different method for the enhancement of the output rf power of the SR BWO. This is based on the same idea, namely, the increase the BWO length together with the increase of the starting threshold. However, instead of a smooth profiling of the electron-wave coupling factor, one proposes its sectioning. In this case, the operating waveguide of the BWO includes two (or more) corrugated interaction sections, which are separated by noncorrugated drift sections, where the electron-wave interaction vanishes. According to simulations, such sectioning can provide approximately the same enhancement of the output rf power as compared to the case of a smooth profiling. However, in sectioned systems the optimal regimes are realized at significantly shorter lengths of the operating region.

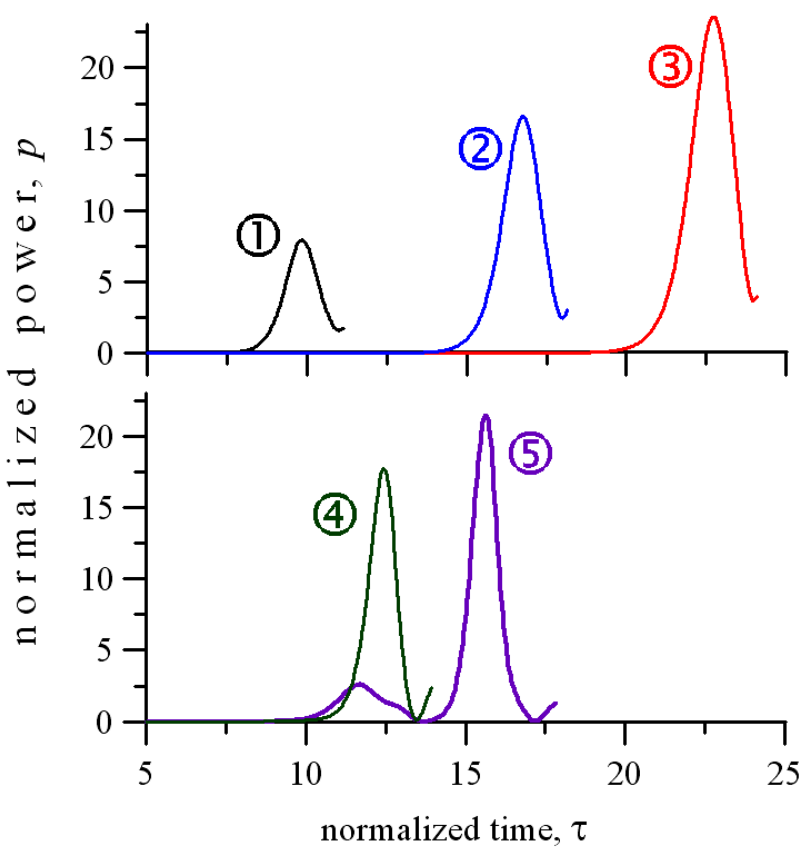

Fig. (5). Normalized power versus the normalized time. Output rf pulses in the cases of the uniform interaction region (1), simple [Eq.(5)] and sectioned [Eq. (6)] linear profiling of the electronwave coupling factor ( 2 and 3 , respectively), as well as in the cases of two-stage and three-stage sectioned systems (4 and 5, respectively).

In the simplest case of two interaction sections,

$\chi(\zeta)= \begin{cases}1, & 0<\zeta<\zeta_{1} \\ 0, & \zeta_{1}<\zeta<\zeta_{2}, \\ 1, & \zeta_{2}<\zeta<L\end{cases}$

the first corrugated section represents an amplifier of the SR rf pulse generated in the second corrugated section (Fig. 6). This system has been optimized by calculating many different parameters $\left(\zeta_{1}, \zeta_{2}, L\right)$ and choosing those yielding the highest peak power of the output rf pulse. According to simulations, in this case of the two-stage sectioned SR BWO the optimal regime takes place at $\zeta_{1}=3.5, \zeta_{2}=5.2$, and the whole normalized length of the system $L=9.5$ (point 4 in Fig. 3), which is comparable with the optimal length of the uniform SR BWO (point 1 in Fig. 3). However, in the sectioned system the output rf power is approximately twice as high $(p \approx 17.7)$. 
Let us notice that the length of the first, "amplifier" section $\left(\zeta_{1}=3.5\right)$ significantly exceeds the starting threshold of self-excitation of this section $\left(L_{\mathrm{st}} \approx 2\right)$. However, this strongly deviates from lengths, which are characteristic for SR regimes of the uniform BWO $(L=7)$. Due to this fact, there is no danger to "spoil" electron beam by a "parasitic" SR rf pulse excited in the first section. At the same time, the second "auto-oscillator" section is significantly longer than the "amplifier" section. Therefore, it is excited faster and generates the SR rf pulse, which is amplified inside the first section.

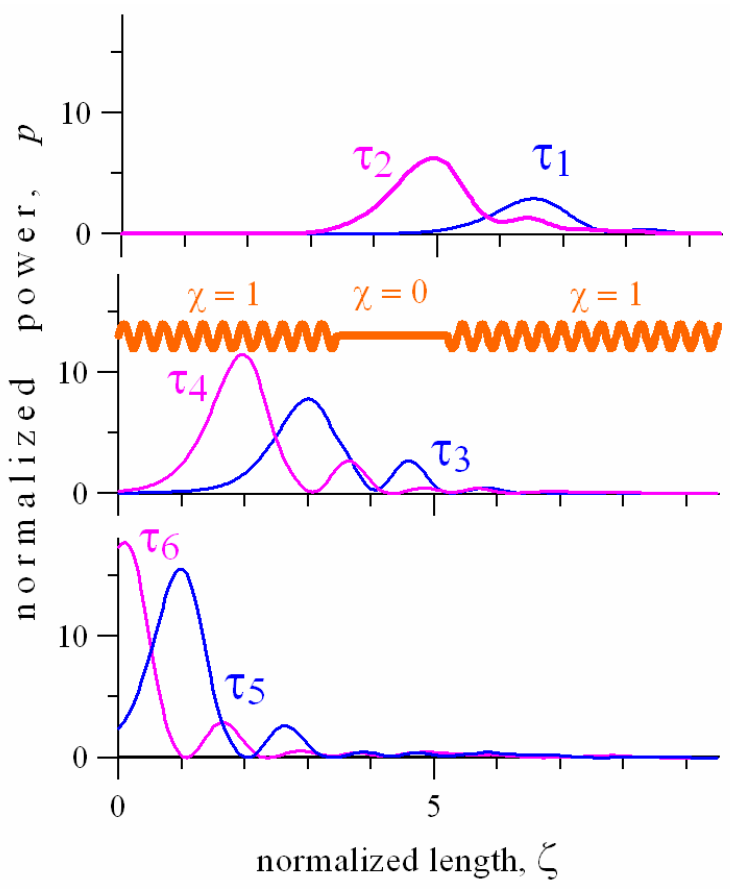

Fig. (6). The two-stage sectioned SR BWO. Spatio-temporal evolution of generation of the SR rf pulse (axial distributions of the normalized $\mathrm{rf}$ power inside the electron-wave interaction region in various moments of the time). The profile of the electron-wave coupling factor, $\chi(\zeta)$, is also shown.

Unfortunately, an additional optimization of this system with two corrugated sections by means of smooth profiling of the electron-wave coupling factor inside the two sections provides no significant improvements. However, there is a possibility to organize a "cascade" amplification of the SR rf pulse in a BWO with three corrugated sections. In this case, the coupling factor has the following form:

$$
\chi(\zeta)= \begin{cases}1, & 0<\zeta<\zeta_{1} \\ 0, & \zeta_{1}<\zeta<\zeta_{2} \\ 1, & \zeta_{2}<\zeta<\zeta_{3} . \\ 0, & \zeta_{3}<\zeta<\zeta_{4} \\ 1, & \zeta_{4}<\zeta<L\end{cases}
$$

In the optimal regime of the cascade SR BWO, the whole length of the system is moderate again (Fig. 3, point 5): $L=12.7 \quad\left(\zeta_{1}=2.9 ; \zeta_{2}=4.5 ; \zeta_{3}=7.5 ; \zeta_{4}=9.1\right)$. This is less than twice longer than the optimal length of the uniform SR BWO; however, as compared to the uniform case, the output $\mathrm{rf}$ power is approximately three times higher $(p \approx 21.5)$.

Shapes of output rf pulses in the optimal regimes of the sectioned SR BWOs are illustrated in Fig. (5) (pulses 4 and 5 , which correspond to two-stage and three-stage operating waveguides, respectively). The behavior of these pulses is very similar to the output rf pulses of the uniform SR BWO, as well as of the SR BWOs with smooth profiling of the electron-wave coupling factor.

\section{CONCLUSION}

The aim of this work is just to point out to a new possible way for optimizing BWOs operating in the regime of generation of SR rf pulses. It is shown that sectioning of the electron-wave interaction region can provide a significant enhancement of the rf power in relatively short systems. The simplest model of the SR BWO is studied in this work, where the asymptotic 2D spatio-temporal equations of the BWO excitations are used to describe an idealized model of the system. This model does not include a number of important effects (for instance, spread in electron velocity, AC and DC space-charge effects, as well as frequency dispersion of the group velocity of the $\mathrm{rf}$ wave). Naturally, all these effects should be taken into account in more detailed simulations of realistic experimental installations.

\section{ACKNOWLEDGMENTS}

The author is very grateful to Dr. E.B. Abubakirov, Prof. N.S. Ginzburg, and Dr. A.S. Sergeev for useful discussions. The work is supported by the Russian Science Support Foundation.

\section{REFERENCES}

[1] Ginzburg NS, Kuznetsov SP, Fedoseeva TN. Theory of transients in relativistic backward-wave tubes. Radiophys Quant Electron 1978; 21: 728-39.

[2] Ginzburg NS, Novozhilova YuV, Zotova IV, et al. Generation of superradiance pulses by high-current subnanosecond electron bunches moving in a periodic slow-wave structure. Tech Phys Lett 1998; 24: 709-11.

[3] Ginzburg NS, Novozhilova YuV., Sergeev AS, et al. Generation of powerful subnanosecond microwave pulses by intense electron bunches moving in a periodic backward wave structure in the superradiative regime. Phys Rev E 1999; 60: 3297-304.

[4] Yalandin MI, Shpak VG, Shunailov SA, Ul'maskulov MR. Experimental study of the transient process in a pulsed relativistic backward-wave tube for the millimeter range. Tech Phys Lett 1999; 25: 388-9.

[5] Elchaninov AA, Korovin SD, Pegel IV, et al. Highly efficient generation of subnanosecond pulses in ka-band and nanosecond pulses in X-band. In: Mehohorn TA, Sweeney MS, Eds. Proc of BEAMS 2002, $14^{\text {th }}$ International conference on high-power particle beams: AIP Conf Proc. Melville, NY 2002; pp. 279-82 vol. 650.

[6] Elchaninov AA, Korovin SD, Rostov VV, et al. Cherenkov Superradiance with a peak power higher than electron flow power. JETP Lett 2003; 77: 266-9. 
[7] Korovin SD, Mesyats GA, Rostov VV, et al. Subnanosecond 1GW pulsed 38-GHz radiation source. Tech Phys Lett 2004; 30: 117-9.

[8] Korovin SD, Elchanonov AA, Rostov VV, et al. Generation of Cherenkov superradiance pulses with a peak power exceeding the power of the driving short electron beam. Phys Rev E 2006; 74 : 016501 .

[9] Ginzburg NS, Korovin SD, Pegel IV, et al. Production of ultrashort high-power microwave pulses in cherenkov backward-wave systems. Laser Phys 2006; 16: 79-88.
[10]

Ginzburg NS, Kuznetsov SP. Periodic and stochastic automodulation regimes in electron RF oscillators with distributed electron-wave coupling, In: Relativistic HF electronics, Issue 2, Institute of Applyed Physics: N.Novgorod, Russia 1989; pp. 10144.

[11] Bratman VL, Ginzburg NS, Petelin MI. Common properties of free electron lasers. Opt Commun1979; 30: 409-12.

(C) A.V. Savilov; Licensee Bentham Open.

This is an open access article licensed under the terms of the Creative Commons Attribution Non-Commercial License (http://creativecommons.org/licenses/by$\mathrm{nc} / 3.0 /$ ) which permits unrestricted, non-commercial use, distribution and reproduction in any medium, provided the work is properly cited. 